\title{
Composición de género en establecimientos escolares chilenos: ¿Afecta el rendimiento académico y el ambiente escolar?
}

\author{
Gender composition in Chilean schools: \\ Does it affect academic achievement and school environment? \\ Composição de género nos estabelecimentos escolares chilenos: \\ afeta o rendimento académico e o ambiente escolar?
Cristóbal Villalobos Dintrans, ${ }^{a}$ Ignacio Wyman San Martín, ${ }^{b}$ Berenice Schiele Muñoz, ${ }^{c}$ Felipe Godoy Ossa ${ }^{d}$ \\ ${ }^{a}$ Centro de Políticas Comparadas de Educación, Universidad Diego Portales \\ Telf.: (56)2676 2236. Correo electrónico: cristobal.villalobos@mail.udp.cl

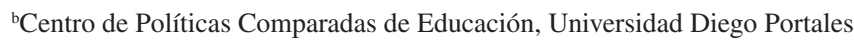 \\ Telf.: (56)2676 2898. Correo electrónico: ignacio.wyman@ cpce.cl \\ 'Telf.: 985011189. Correo electrónico: bmschiel@uc.cl \\ ${ }^{\mathrm{d}}$ Centro de Políticas Comparadas de Educación, Universidad Diego Portales \\ Telf.: (56)2676 2293. Correo electrónico: felipe.godoy@cpce.cl
}

\begin{abstract}
RESUMEN
El estudio analiza las diferencias en rendimiento académico y el ambiente social escolar de una característica particular de la composición de los establecimientos: el género de sus estudiantes. Considerando la discusión sobre eficiencia y eficacia de establecimientos mixtos y no mixtos en Norteamérica y Europa, la investigación analiza los efectos en aspectos académicos y sociales de establecimientos con distinta composición de género en Chile. Para analizar las diferencias netas de la composición de género, se realiza un modelamiento mediante Propensity Score Matching (PSM). Los resultados indican que no existen diferencias significativas en rendimiento académico y convivencia escolar entre establecimientos mixtos y no mixtos en distintos grados (4to Básico, 8vo Básico y 2do Medio), aunque sí se aprecian diferencias estadísticas en algunos grados en autoestima académica a favor de los establecimientos no mixtos. Este conjunto de evidencia plantea nuevos desafíos para las políticas educativas nacionales y la investigación en el área.
\end{abstract}

Palabras clave: eficacia escolar, composición de género, escuelas mixtas, escuelas no mixtas.

\section{ABSTRACT}

This study analyzes the differences in academic achievement and school environment of a special feature of school composition: the gender of the students. Regarding the discussion about efficiency and effectiveness in co-educational and single-sex schools in the USA and Europe, this paper examines the effects on academic and social outcomes of schools with different gender composition in Chile. To analyze net differences of gender composition, a Propensity Score Matching (PSM) method was used. The results show no statistical differences in academic achievement and school environment between co-education and single-sex schools in different grades $\left(4^{\text {th }}, 8^{\text {th }}\right.$ in primary and $10^{\text {th }}$ in secondary), although we found statistical differences in some grades in academic self-esteem in favor of single-sex schools. This evidence suggests new challenges to the national educational policy and future research in this area.

Key words: school effectiveness, gender composition, co-education schools, single-sex schools.

\footnotetext{
La producción de este artículo fue posible gracias al aporte de los proyectos FONDECYT Regular ํ 1150261 y N 1150603.
} 
Estudios Pedagógicos XLII, N 2: 379-394, 2016

COMPOSICIÓN DE GÉNERO EN ESTABLECIMIENTOS ESCOLARES CHILENOS: ¿AFECTA EL RENDIMIENTO

ACADÉMICO Y EL AMBIENTE ESCOLAR?

\section{INTRODUCCIÓN}

La composición escolar ha sido un tema ampliamente abordado en las últimas décadas. En general, se ha utilizado el término composición para denominar a las características agregadas de los miembros que componen un establecimiento educacional, y que se relacionan con el status socioeconómico, el capital cultural, la raza, el género o el rendimiento de los estudiantes (Dupriez, 2010).

Comúnmente, se reconoce como problemático el hecho de que la composición de las escuelas no reflejen la heterogeneidad de las sociedades, sobre todo considerando el rol histórico de socialización del espacio escolar (Durkheim, 1975) y la importancia de la escuela en la producción tanto de procesos de movilidad como de desigualdad social (Bourdieu y Passeron, 1964, 1984; Hansen, 1997). Durante las últimas décadas, se ha desarrollado un intenso debate en torno a la composición escolar. En general, la discusión se ha centrado sobre la relación entre la composición escolar y el logro académico (Dumay y Dupriez, 2008), la magnitud e importancia del efecto de los pares (Thrupp, Lauder y Robinson, 2002; Van Ewijk y Sleegers, 2010; Vandenberghe, 2002) y los efectos composicionales que se producirían en distintos resultados educativos (Opdenakker y Van Damme, 2001; Willms, 2010).

Académicamente, se reconoce que el principal problema en la discusión sobre la composición escolar es que las escuelas deben lidiar (teóricamente) con dos requerimientos a veces contradictorios: deben facilitar a los alumnos el desarrollo de acuerdo a sus aptitudes, pero, al mismo tiempo, deben promover la integración social, instituyendo un set común de valores y conocimientos para enfrentar la vida una vez fuera de la escuela (Dupriez, 2010). La evidencia europea al respecto tiende a destacar que ambos requerimientos suelen manejarse como un trade off. Así, los métodos para maximizar resultados académicos tienden a ser menos inclusivos y generar aulas con características adscritas muy homogéneas (Dupriez, Dumay y Vause, 2008). Por ello, no extraña que países con sistemas escolares selectivos - es decir, colegios que dividen a sus alumnos a temprana edad de acuerdo a sus capacidades - tengan mejores rendimientos promedios pero mayores niveles de inequidad (Duru-Bellat y Suchaut, 2005).

En Chile, los estudios referidos a la composición escolar se han enfocado especialmente en las diferencias en los resultados académicos de acuerdo a la composición socioeconómica de los establecimientos (Bellei, 2007; Drago y Paredes, 2011; Mizala y Torche, 2012), motivados por los altos niveles de segregación socioeconómica de los estudiantes entre escuelas (Valenzuela, Bellei y De Los Ríos, 2014; Villalobos y Valenzuela, 2012). Sin embargo, poco se ha estudiado el efecto que otras variables asociadas a la composición tienen en el desarrollo educativo. Una de estas variables menos abordadas, que es el foco del presente estudio, es el género.

La discusión por la pertinencia del agrupamiento de hombres y mujeres en un mismo establecimiento se aleja cada vez más de argumentos tradicionalistas y valóricos. Actualmente, la pregunta por la composición por género se centra en dilucidar la eficacia de esta política en los resultados académicos, y en menor medida, en el efecto que asistir a un colegio mixto o no mixto podría tener en el ambiente social escolar. Considerando lo anterior, el propósito del presente estudio es identificar el efecto que tiene asistir a un tipo u otro de establecimiento (mixto frente a no mixto) en tres variables: el rendimiento académico, la convivencia escolar y la autoestima académica. De esta forma, se buscará estudiar el efecto 
composicional que el agrupamiento diferenciado por género tendría en distintos aspectos (cognitivos, subjetivos e interrelacionales) relevantes en el proceso escolar.

Para desarrollar este objetivo, el artículo se estructura en cinco apartados, incluyendo esta introducción. En el segundo apartado, se presentan los principales antecedentes de la investigación, dando cuenta de la evidencia nacional e internacional sobre el efecto del agrupamiento por género en los establecimientos escolares. En el tercer apartado se da cuenta de la metodología de investigación utilizada, las fuentes de datos y tratamiento de variables. Los resultados se exponen en el cuarto apartado, donde se explicitan los resultados descriptivos e inferenciales que buscan responder a la pregunta de investigación. Finalmente, el quinto apartado desliza algunas conclusiones, que discuten el rol de la composición escolar en la heterogeneidad escolar, la inclusión social y la eficacia escolar.

\section{ANTECEDENTES}

La discusión sobre el efecto composicional del género está actualmente abierta. Por cuestiones históricas, valóricas y tradicionales, la educación de hombres y mujeres fue, por muchos años, concebida exclusivamente desde la segregación, aludiendo a supuestas diferencias innatas y roles diferenciados que debía reconocer la labor educativa (Silva-Peña, 2012). Sin embargo, desde inicios del siglo XX comienza a abandonarse la separación por género a favor de la coeducación, entendida como la convivencia de hombres y mujeres en el espacio educativo (Subirats, 1994). Desde mediados de los años setenta, y con el aumento sostenido de la competencia en los sistemas escolares de todo el mundo (Apple, 1986; Ball, 2012; Ball y Míguez, 1989), la discusión sobre la composición del género de las escuelas vuelve a ser tematizada, ahora desde la lógica de la eficacia y la eficiencia escolar.

En la actualidad, existen argumentos a favor y en contra de la coeducación. Por una parte, se indica que la coeducación se constituiría como una forma de enseñanza y educación similar a la familia, a la forma natural en que debiesen organizarse las relaciones entre los géneros, permitiendo así la complementariedad y el conocimiento mutuo. Además, se ha argumentado que la coeducación sería la mejor forma de desenvolvimiento de las particularidades específicas de ambos géneros, en la medida en que la identidad de unos no es posible concebirse a distancia de la interacción con el otro (Von Martial y AlvarezValdés, 1992). Finalmente, se ha indicado que la coeducación ofrecería ventajas económicas y organizativas para el núcleo familiar, en la medida en que la posibilidad de mantener a los hijos en un mismo establecimiento, independiente del género de ellos, facilitaría, entre otras cosas, la inclusión de los padres en el proceso educativo de los hijos (March, 2009).

Por otra parte, también se han destacado los posibles factores negativos de la coeducación. Por un lado, se argumenta que la coeducación no se hace cargo de las diferencias biológicas y actitudinales entre hombres y mujeres. Por el contrario, los colegios exclusivos para un solo género favorecerían procesos de aprendizajes más efectivos, en la medida en que muchos de estos establecimientos desarrollan currículos académicos focalizados para hombres o mujeres (Benders, 2012). Otros autores han argumentado que el ambiente coeducativo trae consigo ciertas implicancias en cuanto al desempeño demostrado en algunas asignaturas en particular. Literatura al respecto demuestra que en ambientes coeducativos las mujeres muestran desempeños comparativamente más bajos en asignaturas como matemáticas y ciencias, lo que no ocurre en establecimientos de un solo género (Lee y Lockheed, 1990). 
Incluso, estas diferencias de rendimiento de asistentes a colegios mixtos fomentarían aún más diferencias que se traducen posteriormente en segregación laboral, sobre todo en áreas como las STEM (Science, Technology, Engineering and Mathematics) en donde la fuerza laboral femenina es comparativamente menor (Pahlke, Hyde y Allison, 2014).

Más allá de las interpretaciones y análisis que puedan derivarse de estas discusiones, es posible reconocer tres ámbitos específicos en los que la literatura educativa ha concentrado la investigación sobre la composición escolar y el género: el rendimiento académico, la convivencia escolar y la autoestima académica.

\subsection{EFECTOS DE LA COMPOSICIÓN DE GÉNERO: RENDIMIENTO ESCOLAR, CONVIVENCIA Y AUTOESTIMA}

La mayoría de la evidencia de diferencias entre establecimientos mixtos y no mixtos se enfoca en el logro o rendimiento académico. Desde el histórico Informe Coleman (1961), se ha señalado que la inclusión en una misma sala de clases de hombres y mujeres resultaría perjudicial en el rendimiento académico, en tanto la interacción entre ambos desviaría el foco de atención de la clase. Actualmente, argumentos de este tipo siguen siendo muy respaldados y utilizados, llevando a que durante las últimas décadas en Estados Unidos y el Reino Unido haya habido un aumento en la promoción de escuelas exclusivas para un solo género (Smyth, 2010).

A pesar de ello, las investigaciones internacionales tienden a encontrar efectos muy bajos en el logro académico producto de la coeducación. Por ejemplo, Bone (1983) concluyó que la composición por género explicaba muy poco del rendimiento de los alumnos británicos, una vez controladas otras características asociadas a este tipo de colegios, como los procesos de selección al ingreso. Hannan et al. (1996) respaldaron esta tesis para el caso de Irlanda, encontrando que no existirían diferencias entre escuelas mixtas y no mixtas, donde cerca de un tercio de establecimientos corresponden a colegios de un solo género. En esta misma línea, el meta-análisis de Smyth (2010) destaca que de un total de 39 estudios revisados, 23 indicaban nulo o insignificante efecto de la diferenciación de establecimientos, 15 indicaban una ventaja de los colegios de un solo género y tan solo 1 estudio sustentaba mejores resultados académicos para asistentes a colegios mixtos.

Un segundo ámbito de análisis es el que relaciona la convivencia escolar y la diferencia de composición de género. Para efectos de este estudio, entenderemos la convivencia escolar como la evaluación del comportamiento, estructuras de rol, convenciones, normas y rutinas que inciden en el establecimiento y mantención de relaciones dentro de una comunidad (Ortega-Ruiz y Mora-Merchán, 1998). En general, la evidencia al respecto indica que los colegios mixtos presentarían un ambiente más proclive al desenvolvimiento social de los alumnos (Marsh, 1991). De esta manera, se ha indicado que los establecimientos mixtos, al permitir el contacto de los géneros de manera constante y fluida, permitirían lograr mejores niveles de respeto y de acuerdo al interior tanto del aula como de la escuela. Al respecto, la percepción que tienen los alumnos sobre el género opuesto en los colegios mixtos tiende a ligarse a discursos menos prejuiciosos y a crear menos pensamientos irracionales frente al otro género (Marsh, 1991), pues en la medida en que la interacción con el género opuesto se naturaliza desde temprana edad, se minimizarían las instancias disruptivas entre hombres y mujeres. Además, existe evidencia norteamericana que relaciona la asistencia a colegios mixtos con la mayor valoración de sus alumnos de las instancias que el establecimiento 
les ofrece para desarrollar otras habilidades interrelacionales, como el fomento a la participación en organizaciones, actividades extra-curriculares y una serie de espacios de encuentro entre estudiantes (Schneider y Coutts, 1982; Schneider, Coutts y Starr, 1988).

Finalmente, una tercera variable relevante de análisis se ha concentrado en el efecto de la autoestima académica. A diferencia de la convivencia, la autoestima académica responde a una dimensión personal que incluye juicios sobre la evaluación de la autovalía y de ciertas competencias percibidas en dimensiones específicas (Broc-Cavero, 2000; Rosenberg, 1989). En general, los estudios tienden a presentar un cierto consenso alrededor de la idea de que los colegios no mixtos presentarían condiciones más proclives al desarrollo de este tipo de cualidades individuales orientadas al desarrollo académico. Así, se destaca que, al menos para las mujeres, la asistencia a colegios no mixtos se asociaría a una mayor confianza en sí mismas y mayor autoestima, toda vez que, según se argumenta, se elimina una serie de presiones sociales impuestas por la presencia del otro género (Guglielmi, 2011). Según estos autores, en la medida en que el ambiente escolar carece de las complejidades propias de un ambiente coeducativo, se eliminarían una serie de proyecciones que tanto hombres como mujeres tienen sobre el comportamiento del género opuesto, y que, a su vez, condiciona la manera en que se relaciones unos y otros.

En esta misma línea, Lee y Lockheed (1990) encontraron diferencias estadísticamente significativas en la declaración de planes educacionales, imagen personal y actitudes académicas en alumnos de colegios de un solo género. Los investigadores enfatizaron en que las diferencias son más claras en el caso de colegios de mujeres que de hombres. Por otro lado, destacaron que las mujeres en colegios mixtos presentan alta percepción respecto a cuestiones como la aceptación social, mientras que las mujeres en colegios segregados tienen alta percepción en competencias académicas y conductas de comportamiento.

\subsection{EVIDENCIAS SOBRE LOS EFECTOS DE LA COEDUCACIÓN EN CHILE}

En Chile, existe escasa evidencia de los efectos de la composición de género (coeducación) en el sistema escolar. Por una parte, Silva-Peña (2012) señala que, en general los colegios segregados por género — cerca del 7\% de la matrícula del año 2007serían, por una parte, de dependencia municipal, correspondiendo a los conocidos "liceos emblemáticos"; y por otra, a establecimientos privados, pertenecientes a congregaciones religiosas, y que también por razones históricas y valóricas, han decidido orientarse por la educación diferenciada. Asimismo, el autor señala que además de estas características, las escuelas no mixtas tienden a presentar mayores procesos de selección, siendo, por ello, escuelas menos inclusivas. La conclusión del artículo es que, más allá de la evidencia empírica, la mantención y permanencia de este tipo de colegios se debe a factores sociales, indicando que los colegios de un solo género cumplirían un rol político en la reproducción de las diferencias entre hombres y mujeres.

Por otro lado, March (2009) se enfoca en el tradicional dilema por las diferencias en el rendimiento académico entre hombres y mujeres reflejado en distintos resultados en pruebas estandarizadas de distintas materias. Los resultados destacan que las diferencias en el rendimiento académico comunes en América Latina (Treviño et al., 2010), que tienden a mostrar que los hombres tienen un mejor desempeño en matemática y las mujeres un mejor desempeño en lenguaje, cambian según la composición de género del establecimiento. Sus análisis muestran que aquellos establecimientos coeducativos beneficiarían a los hombres, 
mientras que los establecimientos de un solo género beneficiarían a las mujeres. Además, análisis de regresión demuestran que la composición escolar es un factor más determinante que el género del profesor para predecir los resultados académicos.

Finalmente, Cabezas (2010) se focaliza exclusivamente en el rendimiento académico a partir de la composición según género en las salas de clase. Su contribución se basa en el análisis de la variación en el rendimiento académico en pruebas estandarizadas (SIMCE) de matemática y lenguaje a medida que aumenta el porcentaje femenino de la composición de un curso. Los resultados de su análisis demuestran que a mayor porcentaje de mujeres al interior de la sala de clases, aumenta el rendimiento académico del curso en su conjunto, favoreciendo así la idea de la coeducación, pero también de la educación de un solo género para el caso de las mujeres. Esto vendría a reforzar la idea de la incidencia del efecto grupal en el rendimiento individual de los alumnos. Además, la autora destaca que existen otros aspectos relacionados a la composición del curso que influirían en el rendimiento de los alumnos, como el modo de aproximación de los profesores hacia el alumnado dependiendo de la composición de género que exista en el aula y que crearía ciertas expectativas de rendimiento, e incluso el género del mismo profesor.

\section{METODOLOGÍA}

\subsection{INSTRUMENTOS}

El presente estudio tiene como objetivo dar cuenta del efecto de la composición según género en resultados académicos y de desarrollo social de los niños y niñas, en las escuelas chilenas. Para esto, se consideraron tres conjunto de variables: el logro académico, la autoestima académica de los alumnos y el clima de convivencia escolar. Para la medición del logro académico, se utilizaron dos variables: el promedio de notas de los establecimientos y los resultados de las pruebas estandarizadas del Sistema Nacional de la Calidad Educativa (SIMCE) para Lenguaje, Matemáticas, Ciencias Naturales e Historia en tres grados distintos (4to, 8vo y 2do Medio). Para la medición de la autoestima académica y la convivencia escolar, se construyeron índices estandarizados usando la metodología de Análisis de Componentes Principales (Jolliffe, 2002) para estos mismos grados, a partir de distintas preguntas de los cuestionarios complementarios aplicados a los estudiantes, padres y apoderados durante la prueba SIMCE de los años 2010 y $2011^{1}$.

\subsection{MUESTRA}

Para diferenciar a los establecimientos entre mixtos y no mixtos se analizó el porcentaje de los estudiantes de cada género que componían el establecimiento desde el Sistema de Información General de los Estudiantes (SIGE). Se consideró de un solo género aquellos que tuvieran más de un $95 \%$ de la matrícula de un solo género, considerando posibles errores de digitación en los datos existentes. Bajo esta clasificación, el porcentaje de establecimientos de un solo género en Chile alcanzaba al 5,42\% del total de establecimientos

\footnotetext{
Información sobre las preguntas de los cuestionarios complementarios para alumnos y apoderados incluidas en la medición de la autoestima académica y convivencia escolar puede ser solicitada a los autores.
} 
para el año 2004 (544 establecimientos), mientras que para el año 2013 eran el 5,1\% del total de escuelas (492 establecimientos).

\subsection{MÉTODO DE ANÁLISIS}

Para responder a la pregunta de investigación, los análisis se organizaron en dos etapas. En una primera etapa, se analizaron las diferencias descriptivas entre los establecimientos mixtos y no mixtos de acuerdo a un set de características relevantes, asociadas al tamaño, dependencia administrativa, nivel socioeconómico, procesos de selección, entre otros, para el año 2013.

La segunda etapa del análisis consistió en identificar el efecto de la composición escolar en el rendimiento, la convivencia y la autoestima escolar. La composición escolar por género no es una característica que se distribuye aleatoriamente entre las escuelas, dado que, como muestra la primera fase de análisis, existen diferencias significativas en diversas variables de interés que están relacionadas, por un lado, con ser o no ser una escuela mixta, y a la vez, con los resultados de los niños. Por ende, es necesario aplicar técnicas cuasi-experimentales para reducir al mínimo el sesgo de selección (Khandker, Koolwal y Samad, 2010). Para este propósito, en este estudio se utiliza el Propensity Score Matching (PSM) (Bernal y Peña, 2011) o emparejamiento por propensión, el cual controla el sesgo de selección utilizando características observables de los grupos que se pretende comparar. En nuestro caso, la manera de controlar estas características observables es construyendo una "probabilidad de ser un establecimiento no mixto" mediante un modelo probit de regresión, y luego asignándola a cada establecimiento (Khandker et al., 2010). Finalmente, se empareja cada establecimiento no mixto con uno mixto que posea características observables similares ${ }^{2}$.

\section{RESULTADOS}

De manera coincidente con Silva-Peña (2012), la Tabla 1 muestra que la proporción de establecimientos de un solo género se ha mantenido constante durante los últimos años, con una pequeña disminución porcentual durante el último periodo. Ahora bien, el porcentaje de este tipo de establecimientos no mixtos bordearía el 4\%, lo que difiere en casi 3 puntos porcentuales a lo calculado por el autor.

Tabla 1. Evolución de los colegios mixtos y no mixtos en Chile

\begin{tabular}{lcccccccc}
\hline Grupo & 2004 & 2005 & 2006 & 2007 & 2008 & 2009 & 2010 & 2011 \\
\hline Mixto (\%) & 95,31 & 95,32 & 95,44 & 95,45 & 95,58 & 95,90 & 95,96 & 96,11 \\
No mixto (\%) & 4,69 & 4,68 & 4,56 & 4,55 & 4,42 & 4,10 & 4,04 & 3,89 \\
\hline Total (N) & 11288 & 11560 & 11671 & 11760 & 11905 & 12081 & 12145 & 12044 \\
\hline
\end{tabular}

Fuente: Elaboración propia a partir del Sistema Información General de Estudiantes (SIGE) años 2004-2011

La estimación de la propensión incluye características institucionales, sociodemográficas, orientación religiosa y procesos de selección de los establecimientos. Para más detalle, consultar con los autores. 
Estudios Pedagógicos XLII, N 2: 379-394, 2016

COMPOSICIÓN DE GÉNERO EN ESTABLECIMIENTOS ESCOLARES CHILENOS: ¿AFECTA EL RENDIMIENTO

ACADÉMICO Y EL AMBIENTE ESCOLAR?

Ahora bien, cuando se compara a los establecimientos mixtos y no mixtos en distintas variables, como su dependencia, grupo socioeconómico, tipo de enseñanza y tamaño del establecimiento, se pueden observar importantes diferencias. La Tabla 2 da cuenta de estas diferencias, utilizando y combinando distintas fuentes de información.

Tabla 2. Características estructurales según tipo de establecimiento

\begin{tabular}{|c|c|c|c|}
\hline & \multicolumn{2}{|c|}{ Tipo de establecimiento } & \multirow{2}{*}{ Total } \\
\hline & Mixto & No Mixto & \\
\hline \multicolumn{4}{|l|}{ Dependencia administrativa } \\
\hline Municipal & $52 \% *$ & $48 \% *$ & $52 \%$ \\
\hline Particular subvencionado & $42 \%$ & $43 \%$ & $42 \%$ \\
\hline Particular pagado & $6 \% *$ & $8 \% *$ & $6 \%$ \\
\hline Observaciones & 9426 & 381 & 9807 \\
\hline \multicolumn{4}{|l|}{ Grupo socioeconómico } \\
\hline Bajo & $32 \% *$ & $12 \% *$ & $31 \%$ \\
\hline Medio-bajo & $35 \% *$ & $28 \% *$ & $34 \%$ \\
\hline Medio & $20 \% *$ & $30 \% *$ & $21 \%$ \\
\hline Medio-alto & $8 \% *$ & $19 \% *$ & $9 \%$ \\
\hline Alto & $5 \% *$ & $10 \% *$ & $5 \%$ \\
\hline Observaciones & 7984 & 402 & 8386 \\
\hline \multicolumn{4}{|l|}{ Tipo de enseñanza } \\
\hline Solo educación básica & $65 \% *$ & $46 \% *$ & $65 \%$ \\
\hline Solo educación media & $8 \% *$ & $17 \% *$ & $8 \%$ \\
\hline Educación básica y media & $27 \% *$ & $37 \% *$ & $27 \%$ \\
\hline Observaciones & 9161 & 492 & 9653 \\
\hline \multicolumn{4}{|l|}{ Tipo de enseñanza media impartida } \\
\hline Científico Humanista & $71 \% *$ & $61 \% *$ & $70 \%$ \\
\hline Técnico Profesional & $19 \% *$ & $30 \% *$ & $20 \%$ \\
\hline Polivalente & $10 \%$ & $9 \%$ & $10 \%$ \\
\hline Observaciones & 3142 & 268 & 3410 \\
\hline \multicolumn{4}{|l|}{ Tamaño del establecimiento } \\
\hline Alumnos por establecimiento & $297 *$ & $498^{*}$ & 306 \\
\hline Alumnos por curso & $23 *$ & $27 *$ & 23 \\
\hline Observaciones & 11576 & 468 & 12044 \\
\hline
\end{tabular}

Fuente: Elaboración propia a partir de SIGE 2011, Rendimiento y MIME 2013.

* Diferencias estadísticamente significativas a $95 \%$ de confianza.

Nota 1: Para el tipo de enseñanza media impartida se excluyen establecimientos que solo imparten educación básica.

Nota 2: Las diferencias entre los números de observaciones obedecen al origen diverso de las fuentes de datos utilizadas. 
Los datos muestran que, en general, los establecimientos no mixtos tienden a agrupar alumnos provenientes de familias con un mejor nivel socioeconómico en comparación a aquellos que asisten a colegios mixtos, lo que tiene su correlato en las diferencias en la dependencia administrativa. Asimismo, es interesante observar que un porcentaje mayor de establecimientos no mixtos tiende a impartir educación media (54\% por sobre el 35\% del resto de los colegios). Esto sugiere que la diferenciación según el género de los alumnos ocurre en los grados más altos de la etapa escolar, y podría estar asociada a la diferenciación por especialidades técnicas y/o una especialización con miras a la inserción laboral.

Como complemento a esta información, la Tabla 3 presenta las diferencias entre los establecimientos mixtos y no mixtos según su proyecto educativo, tipos de selectividad (Godoy, Salazar y Treviño, 2014) y orientación religiosa, esto es, algunas características que dan cuenta de las características más "intrínsecas” del establecimiento.

Tabla 3. Presencia de características correspondientes al proyecto educativo, selectividad y religiosidad según tipo de establecimiento

\begin{tabular}{lccc}
\hline & \multicolumn{2}{c}{ Tipo de establecimiento } & \multirow{2}{*}{ Total } \\
\cline { 2 - 3 } & Mixto & No Mixto & \\
\hline Proyecto educativo & & & \\
Excelencia académica & $37 \%$ & $40 \%$ & $38 \%$ \\
Desarrollo integral & $94 \%$ & $96 \%$ & $94 \%$ \\
$\quad$ Enfoque valórico & $12 \% *$ & $34 \% *$ & $12 \%$ \\
Observaciones & 4640 & 190 & 4830 \\
\hline Selectividad & & & \\
$\quad$ Selección según habilidades académicas & $50 \% *$ & $63 \% *$ & $50 \%$ \\
$\quad$ Selección según características familiares & $61 \% *$ & $72 \% *$ & $61 \%$ \\
$\quad$ Observaciones & 9469 & 408 & 9877 \\
\hline Religiosidad & & & \\
Católico & $39 \% *$ & $59 \% *$ & $40 \%$ \\
$\quad$ Otro & $11 \% *$ & $4 \% *$ & $11 \%$ \\
Laico & $50 \% *$ & $37 \% *$ & $49 \%$ \\
Observaciones & 9469 & 408 & 9877 \\
\hline
\end{tabular}

Fuente: Elaboración propia a partir de MIME 2013.

* Diferencias significativas a $95 \%$ de confianza.

En el caso de los énfasis de proyectos educativos, se han incluido las tres características con más presencia en los establecimientos nacionales, según estudios previos (Villalobos y Salazar, 2014). Los resultados muestran que no existen mayores diferencias entre establecimientos mixtos y no mixtos, con excepción de aquellos que tienen enfoque religioso, lo que está en sintonía con los mayores niveles de establecimientos católicos que 
Estudios Pedagógicos XLII, Nº 2: 379-394, 2016

COMPOSICIÓN DE GÉNERO EN ESTABLECIMIENTOS ESCOLARES CHILENOS: ¿AFECTA EL RENDIMIENTO

ACADÉMICO Y EL AMBIENTE ESCOLAR?

existirían en los colegios no mixtos. Por otra parte, al igual que la evidencia internacional, los datos para Chile indican que los colegios no mixtos tienden a ser más selectivos en sus procesos de admisión, ya sea según características familiares como por habilidades de los estudiantes (Smyth, 2010).

En síntesis, los resultados muestran la existencia de importantes diferencias entre establecimientos mixtos y no mixtos en el caso chileno. A continuación, en las tablas 4 y 5 se analizan las diferencias que existen entre ambos grupos, en distintas variables académicas y de ambiente escolar, y que pueden ser atribuidas exclusivamente a la distinta composición de género de los colegios.

Como se puede observar en la Tabla 4, al controlar el sesgo de selección producido por las variables expuestas arriba, no existen diferencias estadísticamente significativas en el rendimiento académico entre establecimientos mixtos y no mixtos para ninguna de las variables utilizadas (SIMCE Lectura, SIMCE Matemática, SIMCE Ciencias Naturales, SIMCE Ciencias Sociales, Promedio Notas), salvo un caso: la diferencia en la prueba de lectura en $8^{\circ}$ básico.

Tabla 4. Diferencias en resultados académicos entre establecimientos mixtos y no mixtos (emparejados)

\begin{tabular}{lcccccccccc}
\hline & \multicolumn{3}{c}{$4^{\circ}$ básico 2011} & \multicolumn{3}{c}{$8^{\circ}$ básico 2011 } & \multicolumn{3}{c}{$2^{\circ}$ medio 2010 } \\
\cline { 2 - 10 } & Mixto & $\begin{array}{c}\text { No } \\
\text { Mixto }\end{array}$ & Dif. & Mixto & $\begin{array}{c}\text { No } \\
\text { Mixto }\end{array}$ & Dif. & Mixto & $\begin{array}{c}\text { No } \\
\text { Mixto }\end{array}$ & Dif. \\
\hline Lectura & 275,9 & 280,4 & 4,5 & 262,3 & 271,1 & $8,8^{*}$ & 271,6 & 275,7 & 4,1 \\
Matemática & 269,6 & 271,1 & 1,5 & 268,5 & 273,1 & 4,6 & 271,7 & 276,6 & 4,9 \\
Cs Naturales & 269,6 & 271,6 & 2 & 273,8 & 278,3 & 4,5 & & & \\
Cs Sociales & & & & 269,9 & 273,8 & 3,9 & & & \\
Promedio notas & 5,9 & 5,9 & $-0,005$ & 5,55 & 5,58 & 0,03 & 5,47 & 5,52 & 0,05 \\
\hline
\end{tabular}

Fuente: Elaboración propia a partir de datos MIME 2013, Rendimiento 2013, SIGE 2011 y SIMCE 2011.

N total de emparejamientos: 4to básico 2011: 178; 8vo básico 2011: 184; 2do medio 2010: 172.

* Diferencias significativas a $95 \%$ de confianza.

Esto implica que, de 12 resultados de rendimiento académico analizados, solo en uno se detectan diferencias a favor de los establecimientos no mixtos. En otras palabras, luego de controlar por aspectos socioeconómicos, académicos y criterios de selección del establecimiento, las diferencias por composición de género desaparecen ${ }^{3}$, tal como ha sugerido distinta evidencia anglosajona (Hannan et al., 1996; Smyth, 2010). De esta forma, serían los arreglos institucionales del establecimiento, junto con otras formas de composición escolar (especialmente la socioeconómica), los que producen las diferencias entre ambos tipos de establecimientos.

Antes del emparejamiento existían diferencias significativas a favor de los establecimientos no mixtos en todas las variables de rendimiento académico analizadas. 
Tabla 5. Diferencias en autoestima académica y clima de convivencia escolar entre establecimientos mixtos y no mixtos (emparejados)

\begin{tabular}{lccccccccccc}
\hline & \multicolumn{3}{c}{$4^{\circ}$ básico 2011} & \multicolumn{3}{c}{$8^{\circ}$ básico 2011} & \multicolumn{3}{c}{$2^{\circ}$ medio 2010} \\
\cline { 2 - 11 } & Mixto & $\begin{array}{c}\text { No } \\
\text { Mixto }\end{array}$ & Dif. & Mixto & $\begin{array}{c}\text { No } \\
\text { Mixto }\end{array}$ & Dif. & Mixto & $\begin{array}{c}\text { No } \\
\text { Mixto }\end{array}$ & Dif. \\
\hline Autoestima académica & 0,69 & 0,79 & 0,11 & 0,17 & 0,27 & 0,1 & 0,3 & 1,04 & $0,74^{*}$ \\
Clima de respeto & $-0,28$ & $-0,14$ & 0,14 & $-0,78$ & $-1,3$ & $-0,53^{*}$ & 0,54 & 0,95 & 0,41 \\
Clima seguro & & & & 0,54 & 0,09 & $-0,45$ & $-0,34$ & $-0,63$ & $-0,29$ \\
Clima organizado & 0,51 & 0,06 & $-0,45^{*}$ & 0,82 & 1,12 & 0,3 & 0,43 & 0,92 & $0,49^{*}$ \\
\hline
\end{tabular}

Fuente: Elaboración propia a partir de datos MIME 2013, Rendimiento 2013, SIGE 2011 y cuestionarios para estudiantes y padres y apoderados SIMCE 2010 y 2011.

Nota: Los valores corresponden a puntajes estandarizados. Se interpretan como la desviación estándar desde la media.

N total de emparejamientos: 4to básico 2011: 168; 8vo básico 2011: 173; 2do medio 2010: 150.

* Diferencias significativas a $95 \%$ de confianza.

La Tabla 5 muestra el análisis de los resultados para las distintas variables de ambiente escolar. En este caso, luego de realizar el emparejamiento, podemos observar que no existen diferencias estadísticamente significativas en ninguna variable para todos los niveles. Así, mientras existe una diferencia a favor de los establecimientos mixtos en la variable de clima organizado en 4to básico, hay una diferencia a favor de los no mixtos en esta misma variable en 2do Medio. Asimismo, tampoco se observa un patrón claro en el caso de la autoestima académica, (que es significativa a favor de los establecimientos no mixtos solo en la medición de 2do Medio), lo que contrasta con los resultados reportados por otros estudios (Lee y Bryk, 1989).

Ahora bien, ¿Son estos resultados extrapolables para el conjunto de los establecimientos? ¿Existen algunos establecimientos donde efectivamente exista un efecto de la composición de género? Para responder a esta pregunta, la Tabla 6 presenta estadística descriptiva que compara algunos resultados académicos (SIMCE Matemática y SIMCE Lectura) y de ambiente social escolar (Autoestima Académica y Clima de Respeto) distinguiendo por la dependencia y grupo socioeconómico, solo para el subconjunto de los establecimientos emparejados. Como se puede observar, los resultados muestran que existen diferencias en resultados académicos entre colegios mixtos y no mixtos en algunos tipos de establecimientos, como los grupos socioeconómicos medios y altos y los particulares subvencionados en Educación Básica y municipal en la Educación Media. Respecto a la autoestima académica, solo existen diferencias significativas entre establecimientos de nivel socioeconómico medio en los resultados de 4to básico. Para el clima de respeto, las diferencias tienden a concentrarse entre establecimientos de niveles medios y particulares subvencionados considerando los índices para 8vo básico. Para la educación media, las diferencias también son significativas a nivel medio. Esto podría estar indicando que el efecto de composición de género podría ser relevante en algunos subgrupos de establecimientos —aunque no en el conjunto de estos-, aún después que se controla por distintas diferencias observables, aunque esta es una hipótesis a testear en próximas investigaciones. 
Estudios Pedagógicos XLII, N 2: 379-394, 2016

COMPOSICIÓN DE GÉNERO EN ESTABLECIMIENTOS ESCOLARES CHILENOS: ¿AFECTA EL RENDIMIENTO ACADÉMICO Y EL AMBIENTE ESCOLAR?

Tabla 6. Diferencias en rendimiento académico en sub-grupos de establecimientos (según nivel socioeconómico y dependencia) emparejados

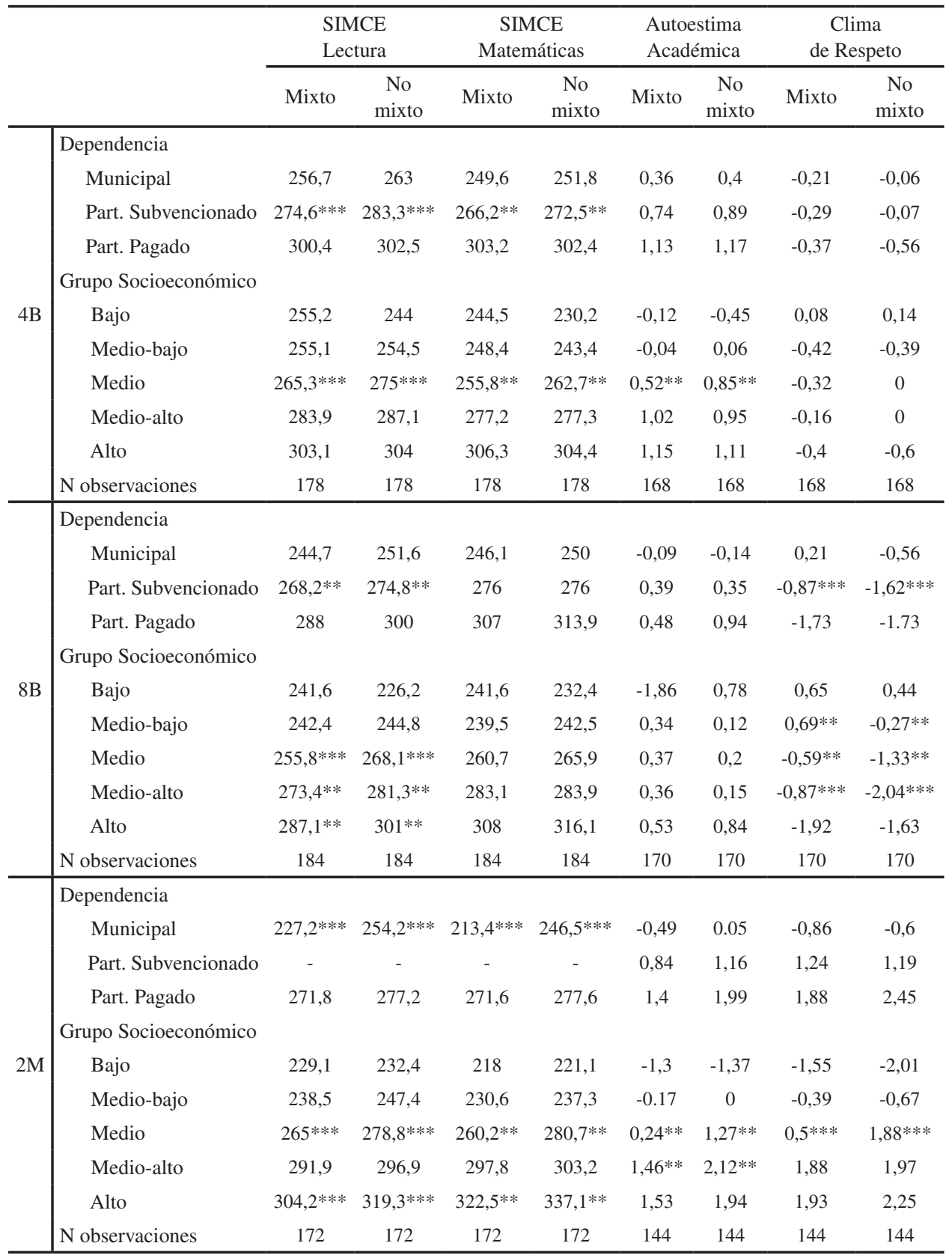

Fuente: Elaboración propia a partir de datos MIME 2013, Rendimiento 2013, SIGE 2011 y cuestionarios para estudiantes y padres SIMCE 2011 y 2010.

$* *$ Diferencias significativas a $95 \%$ de confianza; $* * *$ Diferencias significativas a $99 \%$ de confianza 


\section{CONCLUSIONES}

Los resultados muestran que la composición de género en Chile no es un factor central en la generación de mayores resultados académicos, niveles de convivencia o autoestima escolar. Asimismo, se ha mostrado que los establecimientos que realizan procesos de segregación de su matrícula por género (establecimientos no mixtos) constituyen una pequeña fracción del conjunto de establecimientos, con características particulares: mayores niveles de selección, niveles socioeconómicos más altos, mayores tamaños y proyectos educativos con énfasis religiosos.

Esta evidencia permite incorporar elementos a la comprensión de los procesos de gestión de la heterogeneidad educativa, inclusión social y segregación escolar, pudiéndose rescatar especialmente tres elementos. En primer lugar, los resultados reflejan que la composición escolar de género puede entenderse como un mecanismo de diferenciación de los estudiantes, en promedio, ineficiente. En el marco de un sistema escolar segregado y profundamente diferenciado como el chileno (Valenzuela et al., 2014; Villalobos y Valenzuela, 2012) esto es relevante, pues lleva a preguntarse por la necesidad o no de mantener este tipo de diferenciaciones, más allá de las preferencias valóricas o las condiciones históricas que sustentan la educación diferenciada entre niños y niñas.

En segundo lugar, y relacionado con lo anterior, la discusión sobre la composición de género en Chile permite relacionar la discusión sobre el efecto del género dentro de la discusión sobre la inclusión escolar. Hasta la fecha, la mayoría de las políticas implementadas o por implementar (MINEDUC, 2014), así como muchos de los estudios o investigaciones realizadas (López et al., 2014; Treviño, Salazar y Donoso, 2011; Valenzuela, 2011) han concentrado la discusión sobre la inclusión en aspectos socioeconómicos o académicos. Los resultados de este estudio son claros al mostrar que la educación exclusiva para un solo género en Chile no genera mejores resultados académicos ni tiene efectos claros en el ambiente social escolar, dando así cuenta de la necesidad de incorporar la variable de género como un componente fundamental de la inclusión educativa. ¿Cómo gestionar los establecimientos para que niños y niñas convivan en un mismo espacio? ¿Qué herramientas y dispositivos pedagógicos se necesitan para que estudiantes de distinto género puedan colaborar y aportar positivamente en su proyecto educativo? Entre otras, preguntas como estas pueden considerarse importantes interrogantes para la política educativa.

Finalmente, parece necesario reflexionar sobre nuevas líneas de investigación que se desprenden de esta investigación. Al respecto, es claro que es necesario incorporar más información sobre la forma en cómo la composición escolar de género es vivenciada por los establecimientos y distintos actores escolares, dando cuenta de las posibles tensiones o contradicciones existentes en estos proceso. Adicionalmente, es necesario estudiar cómo otras variables o resultados escolares (trayectorias académicas, deserción escolar, desarrollo de habilidades sociales, habilidades tecnológicas) tienen efectos (o no) de manera diferenciada en los establecimientos mixtos o no mixtos, profundizando así en el conocimiento de la composición escolar de género en el país. 
Estudios Pedagógicos XLII, $\mathrm{N}^{\circ}$ 2: 379-394, 2016

COMPOSICIÓN DE GÉNERO EN ESTABLECIMIENTOS ESCOLARES CHILENOS: ¿AFECTA EL RENDIMIENTO

ACADÉMICO Y EL AMBIENTE ESCOLAR?

\section{REFERENCIAS BIBLIOGRÁFICAS}

Apple, M. (1986). Ideología y currículo (Vol. 101). Madrid: Ediciones Akal.

Ball, S. (2012). Privatizaçoa da educaçao e novas subjetividades: contornos e desdobramientos das políticas (pós) neoliberais. Revista Brasileira de Educação, 18(53), 457-466.

Ball, S., \& Míguez, N. (1989). La micropolítica de la escuela: hacia una teoría de la organización escolar. Barcelona: Paidós.

Bellei, C. (2007). Expansión de la educación privada y mejoramiento de la educación en Chile. Evaluación a partir de la evidencia. Expansion of private schools and educational improvement in Chile. An evidence-based evaluation. Revista Pensamiento Educativo, 40(1), 1-37.

Benders, D. S. (2012). A Review of Gender-Segregated Classrooms in Public Schools. Recuperado desde: http://ssrn.com/abstract=2011781.

Bernal, R., \& Peña, X. (2011). Guía práctica para la evaluación de impacto. Santiago de Chile: Centro de Estudios sobre Desarrollo Económico, Facultad de Economía, Universidad de los Andes.

Bone, A. (1983). Girls and Girl-only School. Manchester: Equal Opportunities Commission.

Bourdieu, P., \& Passeron, J. C. (1964). Los herederos. Los estudiantes y la cultura. Buenos Aires: Siglo Veintinuo Ediciones.

Bourdieu, P., \& Passeron, J. C. (1984). La reproducción. Elementos para una teoría de la enseñanza. Madrid: Popular.

Broc-Cavero, M. Á. (2000). Autoconcepto, Autoestima y Rendimiento en alumnos de $4^{\circ}$ de ESO Implicaciones Psicopedagógicas en la Orientación y Tutoría. Revista de Investigación, 18(1), 119-146. doi:http://dx.doi.org/10.6018/rie.18.1.121521

Cabezas, V. (2010). Gender Peer Effects in School: Does the Gender of School Peers Affect Student Achievement? (Doctoral Dissertation). Columbia University.

Coleman, J. (1961). The adolescent society. The social life of the teenager and its impact on education. EE.UU.: Free Press of Glenco.

Drago, J. L., \& Paredes, R. (2011). La brecha de la calidad en la educación chilena. CEPAL Review, 104.

Dumay, X., \& Dupriez, V. (2008). Does the school composition effect matter? Evidence from Belgian data. British Journal of Educational Studies, 56(4), 440-477. doi:10.1111/j.14678527.2008.00418.x

Dupriez, V. (2010). Methods of Grouping Learners at School (Vol. 93). París: UNESCO: International Institute for Educational Planning.

Dupriez, V., Dumay, X., \& Vause, A. (2008). How Do School Systems Manage Pupils' Heterogeneity? Comparative Educational Review, 52(2), 245-273. doi:10.1086/528764

Durkheim, E. (1975). Educación y sociología. Barcelona: Editorial Península.

Duru-Bellat, M., \& Suchaut, B. (2005). L'approche sociologique des effets du contexte scolaire: Méthodes et difficultés. Revue internationale de psychologie sociale, 18(3), 5-42.

Godoy, F., Salazar, F., \& Treviño, E. (2014). Prácticas de selección en el sistema escolar chileno: tipos de requisitos de postulación, legitimación en el sistema y las debilidades de la ley. Informes para la Política Educativa $N^{o}$ 2. Santiago: Centro de Políticas Comparadas de Educación, Universidad Diego Portales.

Guglielmi, A. (2011). Girls' Self-Esteem Rates in Single Sex \& Coed High Schools. Recuperado desde: http://digitalrepository.trincoll.edu/theses/52

Hannan, D., Smyth, E., McCullagh, J., O'Leary, R., \& McMahon, D. (1996). Coeducation and gender equality: exam performance, stress and personal development. Dublin: Oak Tree Press.

Hansen, M. (1997). Social and Economic Inequality in the Educational Career: Do the Effects of Social Background Characteristics Decline? European Sociology Review, 13(3), 305-321.

Jolliffe, I. (2002). Principal Component Analysis. England: Springer. 
Khandker, S., Koolwal, G., \& Samad, H. (2010). Handbook on impact evaluation: quantitative methods and practices. Washington: World Bank Publications.

Lee, V., \& Bryk, A. (1989). Effects of single-sex schools: Response to Marsh. Journal of Educational Psychology, 81(4), 647-650. doi:10.1037/0022-0663.81.4.647

Lee, V., \& Lockheed, M. (1990). The effects of single-sex schooling on achievement and attitudes in Nigeria. Comparative Education Review, 34(2), 209-231.

López, V., Maturana, C., Morales, M., Rojas, C., \& Villalobos, M. (2014). Barreras culturales para la Inclusión: políticas y prácticas de Integración en Chile. Revista de educación, 363, 256-281. doi:10.4438/1988-592X-RE-2012-363-180

March, D. (2009). Diferencias de género en rendimiento académico: efectos en la evolución a nivel escolar (Memoria para optar el título de ingeniero civil en industrias). Pontificia Universidad Católica de Chile.

Marsh, H. W. (1991). Public, Catholic single-sex, and Catholic coeducational high schools: Their effects on achievement, affect, and behaviors. American journal of education, 99(3), 320-356.

MINEDUC. (2014). Una nueva educación para Chile. Documento base para los diálogos temáticos Plan Nacional de Participación Ciudadana. Santiago: Autor.

Mizala, A., \& Torche, F. (2012). Bringing the schools back in: the stratification of educational achievement in the Chilean voucher system. International Journal of Educational Development, 32(1), 132-144. doi:10.1016/j.ijedudev.2010.09.004

Opdenakker, M.C., \& Van Damme, J. (2001). Relationship between School Composition and Charactertiristics of School Process and their Effect on Mathematics Achievement. British Educational Research Journal, 27(4), 407-432. doi:10.1080/0141192012007143 4

Ortega-Ruiz, R., \& Mora-Merchán, J. (1998). La convivencia escolar: qué es y cómo abordarla. Programa Educativo de prevención de maltrato entre compañeros y compañeras. España: Consejería de Educación y Ciencia Junta de Andalucía.

Pahlke, E., Hyde, J., \& Allison, C. (2014). The Effects of Single-Sex Compared With Coeducational Schooling on Students' Performance and Attitudes: A Meta-Analysis. Psychological Bulletin, 140(4), 1042-1072.

Rosenberg, M. (1989). Society and the adolescent self-image (rev. ed.). Middletown: Wesleyan University Press.

Schneider, F., \& Coutts, L. (1982). The high school environment: A comparison of coeducational and single-sex schools. Journal of Educational Psychology, 74(6), 898-906. doi:10.1037/00220663.74.6.898

Schneider, F., Coutts, L., \& Starr, M. (1988). In favour of coeducation: The educational attitudes of students from coeducational and single-sex high schools. Canadian Journal of Education/Revue canadienne de l'education, 13(4), 479-496.

Silva-Peña, I. (2012). Repensando la escuela desde la coeducación. Una mirada desde Chile. Revista Venezolana de Estudios de la Mujer, 15(35), 161-176.

Smyth, E. (2010). Single-sex education: What does Research Tell us? Revue française de pédagogie, $171(2), 47-58$.

Subirats, M. (1994). Conquistar la igualdad: la coeducación hoy. Revista Iberoamericana de Educación, 6, 49-78.

Thrupp, M., Lauder, H., \& Robinson, T. (2002). School composition and peer effects. International journal of educational research, 37(5), 483-504. doi:10.1016/S0883-0355(03)00016-8

Treviño, E., Salazar, F., \& Donoso, F. (2011). ¿Segregar o incluir? Esa no debería ser una pregunta en educación. Revista Docencia, 45(1), 34-47.

Treviño, E., Valdés, H., Castro, M., Costilla, R., Pardo, C., \& Donoso, F. (2010). Factores asociados al logro cognitivo de los estudiantes de América Latina y el Caribe. Santiago: SERCE/UNESCO.

Valenzuela, J. P. (2011). La gratuidad como derecho y condición para una educación de calidad para todos. Revista Docencia, 45(1), 18-24. 
Valenzuela, J. P., Bellei, C., \& De Los Ríos, D. (2014). Socioeconomic school segregation in a market-oriented educational system. The case of Chile. Journal of Education Policy, 29(2), 217241. doi:10.1080/02680939.2013.806995

Van Ewijk, R., \& Sleegers, P. (2010). The effect of peer socioeconomic status on student achievement: A meta-analysis. Educational Research Review, 5(2), 134-150. doi: 10.1016/j.edurev.2010.02.001

Vandenberghe, V. (2002). Evaluating the magnitude and the stakes of peer effects analysing science and math achievement across OECD. Applied Economics, 34(10), 1283-1290. doi:10.1080/00036840110094446

Villalobos, C., \& Salazar, F. (2014). Proyectos educativos en el sistema escolar chileno: una aproximación a las libertades de enseñanza y elección. Informe para la Política Educativa $N^{o} 2$. Santiago: Centro de Políticas Comparadas en Educación.

Villalobos, C., \& Valenzuela, J. P. (2012). Polarización y Cohesión social del Sistema Escolar Chileno. Revista de Análisis Económico, 27(2), 145-172.

Von Martial, I., \& Alvarez-Valdés, M. (1992). Coeducación: ventajas, problemas e inconvenientes de los colegios mixtos. España: Ediciones Universidad de Navarra. EUNSA.

Willms, J. D. (2010). School composition and contextual effects on student outcomes. The Teachers College Record, 112(4), 3-4. 